\section{Attacking the sleeping sickness parasite}

\section{By Tim Fulmer, Senior Writer}

U.K. researchers have designed a small molecule inhibitor of trypanosome N-myristoyltransferase, an enzyme necessary for survival of the parasite that causes sleeping sickness. The compound cured two different mouse models of blood-stage African trypanosomiasis, ${ }^{1}$ but will now require optimization to ensure it can cross the blood brain barrier to treat the more deadly CNS forms of the disease.

African trypanosomiasis (sleeping sickness) is caused by Trypanosoma brucei, a protozoan that is transmitted to humans by the bite of tsetse flies. After infection by a fly bite, the parasites first proliferate in the blood and lymphatic systems (stage one) before invading CNS tissue (stage two). At this stage, infection leads to progressive mental deterioration and serious sleep cycle disruptions.

Because stage-one disease is characterized by nonspecific symptoms such as malaise and fever, sleeping sickness can often go undiagnosed until the serious neurological symptoms of stage-two infection become apparent. At that point, the disease is typically fatal without treatment.

Four drugs are approved to treat sleeping sickness, although their modes of action are generally unclear. ${ }^{2}$ Two of these drugs are effective against stage-two disease but have significant drawbacks. Melarsoprol, a generic arsenic derivative, has severe side effects and a 5\% treatment-related mortality rate. The generic eflornithine, though less toxic, requires a complex intravenous treatment regimen that is often impractical in developing areas where skilled medical staff are in short supply.

Thus, there is a need for orally available trypanosome-selective therapies that treat stage-two disease.

The new findings by a team led by Paul Wyatt, professor of biological chemistry and drug discovery at the University of Dundee, and Deborah Smith, professor of molecular parasitology at the University of York, builds on work by their own labs and others that suggests that Trypanosoma brucei N-myristoyltransferase (NMT; TbNMT; Tb10.61.2550) could be a good trypanosome target.

NMT catalyzes the transfer of myristic acid, a 14-carbon fatty acid, to the N-terminal glycine residue of a variety of proteins. The hydrophobic myristic acid then allows proteins to localize to the membrane to carry out their cellular functions. Inhibiting NMT thus should impair the function of all proteins that require the myristate modification, leading to dysfunction of multiple cellular pathways and cell death.

Previously, Smith and colleagues used RNA knockdown to confirm that TbNMT was required for trypanosome growth, ${ }^{3,4}$ and NMT inhibitors designed for fungal species showed activity against cultured T. brucei. ${ }^{5}$ The open question was whether it would be possible to design inhibitors of TbNMT that did not also inhibit mammalian NMT.

To help answer that question, Smith teamed up with Wyatt, who is director of Dundee's Drug Discovery Unit and head of the university's Discovery for Tropical Diseases initiative.

The team screened a 62,000-compound library against TbNMT and identified a pyrazole sulfonamide derivative with moderate potency against the enzyme. Subsequent optimization of that hit, which involved designing and synthesizing more than 200 compounds, eventually led to a series of TbNMT inhibitors.

The most potent compound was designated DDD85646. In a mouse model of acute sleeping sickness infection, it cured all mice of infection at a minimal oral dose of $12.5 \mathrm{mg} / \mathrm{kg}$ twice a day for four days.

Delivering DDD85646 at higher doses using shorter dosing schedules-100 mg/kg twice a day for one day and $25 \mathrm{mg} / \mathrm{kg}$ twice a day for two days-also cured all treated mice.

In a second mouse model of a more refractory form of acute sleeping sickness, DDD85646 again cured all mice of infection at a minimal dose of 50 $\mathrm{mg} / \mathrm{kg}$ twice a day for two days.

The compound was well tolerated. Results were published in Nature.

\section{Approaching optimal}

Compared with existing drugs, DDD85646 and its analogs "have the advantage that they can be orally delivered, have a defined target in the parasite, and could potentially show good specificity over the target's human counterpart," said Keith Matthews, professor of parasite biology at the University of Edinburgh.

To get some initial hints about safety in humans, the researchers could next "demonstrate that the inhibitors are nontoxic in cultured primary human cells at the doses they used to treat the blood-stage infection," said Elena Gonzalez-Rey, professor of cellular biology and immunology in the López-Neyra Institute of Parasitology and Biomedicine at the Granada Health Science Technological Park.

"It would also be standard preclinical procedure to develop toxicological profiles of these inhibitors in two mammalian species-for example, rodents and dogs, or rodents and primates-and in particular to look for any signs of renal and hepatic toxicity," said Richard Tidwell, 


\section{ANALYSIS}

\section{TARGETS \& MECHANISMS}

professor of pathology and laboratory medicine at the University of North Carolina at Chapel Hill and director of the Consortium for Parasitic Drug Development (CPDD).

Matthews also said it will be important to perform any further chemical optimization necessary to allow the inhibitors to cross the blood brain barrier into the CNS, because "it is parasite in the CNS that generates the neurological pathology that ultimately leads to death."

Indeed, the therapeutic value of the compounds may stand or fall solely on their ability to get into the CNS.

"It's a fundamental question whether compounds that are active against only early stage trypanosomiasis, before brain involvement, are actually even required," said Michael Barrett, professor of parasitology at the University of Glasgow.

He noted that according to criteria published by the World Health Organization (WHO), the Drugs for Neglected Diseases Initiative (DNDi) and CPDD, any new sleeping sickness therapies should be able to target and treat late-stage disease with CNS involvement.

In the Nature paper, the authors state that DDD85646 "does not yet meet these criteria."

Thus, Barrett said it will be necessary to alter the chemical structures of the Nature compounds "to enable them to penetrate the blood brain barrier, while retaining good efficacy against the parasites."

Wyatt agreed his team must now optimize the inhibitors to enable them to treat late-stage sleeping sickness infection with CNS involvement.

"We have already made some progress towards that goal starting from the compounds mentioned in the paper, but we still have some work to do," he said. "Because we have a significant understanding of what is required to help us achieve the desired molecular properties, we are hoping we can identify a preclinical candidate within the next year."
Wyatt added, "Once we have an optimized compound, we will work with the Drugs for Neglected Diseases Initiative to help carry out the preclinical and clinical development."

DNDi is a not-for-profit organization that funds early stage research to identify new compounds to treat sleeping sickness and other neglected diseases. The organization also funds clinical trials. Under a 2008 deal, the Dundee group and DNDi are screening for new compounds to treat leishmaniasis and sleeping sickness.

Wyatt and colleagues will continue to investigate the therapeutic potential of other targets in T. brucei.

The inhibitors described in the Nature paper are covered by patents and are available for licensing.

Fulmer, T. SciBX 3(16); doi:10.1038/scibx.2010.485

Published online April 22, 2010

\section{REFERENCES}

1. Frearson, J. et al. Nature; published online April 1, 2010; doi:10.1038/ nature08893

Contact: Paul Wyatt, University of Dundee, U.K. e-mail: p.g.wyatt@dundee.ac.uk

2. Barrett, M. et al. Br. J. Pharmacol. 152, 1155-1171 (2007)

3. Price, H. et al. J. Biol. Chem. 278, 7206-7214 (2003)

4. Price, H. et al. Mol. Biochem. Parasitol. 169, 55-58 (2010)

5. Panethymitaki, C. et al. Biochem. J. 396, 277-285 (2006)

\section{COMPANIES \& INSTITUTIONS MENTIONED}

Consortium for Parasitic Drug Development (CPDD), Chapel Hill, N.C. Drugs for Neglected Diseases Initiative (DNDi), Geneva, Switzerland Granada Health Science Technological Park, Armilla, Spain University of Dundee, Dundee, U.K. University of Edinburgh, Edinburgh, U.K. University of Glasgow, Glasgow, U.K.

University of North Carolina at Chapel Hill, Chapel Hill, N.C.

University of York, Heslington, U.K.

World Health Organization (WHO), Geneva, Switzerland 
